\title{
Analyzing runs of an event occurring in a series with other kinds of events
}

\author{
BRUCE DORVAL \\ University of New Orleans, Lakefront, New Orleans, Louisiana
}

\begin{abstract}
A method for analyzing runs of an event occurring in a series with other kinds of events is presented. The first step of the analysis is to determine whether the event of interest occurs in longer runs than would be expected by chance. For this purpose, a cumulative frequency histogram, consisting of the relative proportions of runs as a function of length, is compared with a theoretically derived frequency histogram by means of the Kolmogorov-Smirnov test. The theoretical histogram is the outcome of a Bernoulli process; it represents the distribution of run lengths that would be expected by chance for a given base-rate probability of occurrence of the event of interest. Iteratively applied $Z$ tests are proposed as a post hoc procedure for use when the Kolmogorov-Smirnov test reveals that the histogram of runs is not comparable to the theoretical histogram. The results reveal how the two histograms differ. Performing the same analysis on the class of all other events reveals whether runs occur in proximity. When they do, the frequency histogram for the event should be recalculated, allowing for brief intervening runs of other events, and tested with the appropriate theoretical distribution.
\end{abstract}

Sequential analysis of observational data is an important research tool for examining the microstructure of social interaction. Such techniques have been used to examine parent-infant interaction (e.g., Als, Tronick, \& Brazelton, 1979; Bakeman \& Brown, 1977; Brownlee \& Germond, 1984; Sackett, 1979), early peer interaction (e.g., Bakeman \& Brownlee, 1982; Eckerman \& Stein, 1982; Goldman \& Ross, 1978), older peer interaction (e.g., Dorval \& Eckerman, 1984; Gottman, 1983; Suomi, 1979), and the interactions of couples and families (e.g., Gottman, 1979; Patterson, 1980). These studies and others demonstrate that sequential analysis is an important means of summarizing complex data and of testing hypotheses pertaining to interactive processes. They demonstrate also that the range of application of sequential-analysis procedures is widening and that new procedures are being developed.

The method of sequential analysis proposed in this paper emerged as a result of using the lag-sequential technique (Allison \& Liker, 1982; Bakeman, 1983; Gottman, 1979; Gottman \& Bakeman, 1979; Sackett, 1977, 1979, 1980) to examine the conversation of small groups of peers. The conversations were transcribed as series of speaking turns (see Dorval \& Eckerman, 1984, pp. 16-17), and each turn was coded according to its relationship with directly preceding turns. The lag-sequential technique revealed that certain kinds of turns display strong autodependency; that is, each is likely to be followed in conversation by another of its kind. When this occurs, runs are constructed

\footnotetext{
I appreciate the Computer Research Center of the University of New Orleans, Lakefront, for donating computer time for this study. I thank Mark Applebaum, Roger Bakeman, and Cameron Camp for helpful comments on earliet drafts of this paper. My mailing address is: Psychology Department, University of New Orleans, Lakefront, New Orleans, LA 70148.
}

differently than would be expected by chance, although the lag-sequential method does not reveal exactly how. This paper reports a method for obtaining such information.

The proposed method is demonstrated on two kinds of turns in one conversation generated by a small group of fifth-grade students. The results reveal that each kind of turn has a distinct run structure and that together they make a substantial contribution to the overall structure of the sample conversation.

\section{DATA DEFINITION PRELIMINARIES}

Sequential data consist of a series of events, with each event assigned one code of a mutually exclusive and exhaustive set. The coding of each event must be independent of the coding of prior or subsequent events. For present purposes, one category of event or grouping of categories is selected as the event of interest $(X)$. By definition, all others are not the event of interest. (These non $X$ events are labeled " $O$ ".) The beginning and ending of a series of data look like the following, after collapsing to $X$ s and $O$ s:

OOOXOOXXOXXX000000XXОХXXX0000000

OOXXOOOOOXOXXOOOOXXOOXXXX.....

ОOОХХОХХХOOОХОООООООООХХХХХООХХ०

OOXXXXОХОOОХХOOOOOXXОХХ

\section{RUNS OF DISAGREEMENTS IN A CONVERSATION GENERATED BY A SMALL GROUP OF FIFTH GRADERS}

Disagreements and story continuations in the same con- 
versation were examined. The run structure of disagreements was determined first.

\section{Step 1: Examining the Distribution of Runs}

The first step in a runs analysis is to determine the baserate probability of occurrence of disagreements. Because we are treating the data as a series of runs, it is appropriate to discard the fragments of runs at the beginning and end of the data stream. Using the above sample of data, the first three $O$ s and last two $X s$ are discarded. Next, the probability of disagreements is computed, by dividing the total number of disagreements by the total number of turns in the trimmed series (.295 for the sample conversation).

This procedure yields an estimate of $p(X)$. Thus, the Kolmogorov-Smirnov test is most appropriately performed on the upper and lower bounds of a confidence interval erected around the estimate, requiring both to be greater than the critical value before the null hypothesis is rejected. Fortunately, when samples are large-a requirement of the proposed method-the standard error of estimate is very low (.014 for the sample conversation) and the confidence interval is correspondingly narrow (95\% confidence interval: .268-.322).

The estimate of $p(X)$ is used to generate the histogram of run lengths that would be expected by chance. This histogram is the result of a Bernoulli process. It is represented in the following tree diagram:

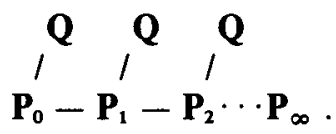

Imagine moving along in the series until $P_{o}$ is encountered. The probability that it is not followed by another $P$ is $Q$. Thus, the probability of a run of Length 1 is $Q$. The probability that $P_{o}$ is followed by just one $P$ is $P * Q$, and that it is followed by just two $P$ s is $P^{2} * Q$. Thus, the probability of runs of Length 2 is $P * Q$, and the probability of runs of Length 3 is $P^{2} * Q$. The model generates an infinite, convergent series that sums to 1 . It may be summarized as follows:

$$
\sum_{i=1, \infty} Q P^{i-1}
$$

The formula was verified by computer modeling. (This and other programs mentioned below are available from the author at no cost.) Drake (1967) proposed essentially the same formula for modeling the distribution of times between the arrival of buses (pp. 126-127).

Panel A of Figure 1 presents the observed and theoretical histograms for the disagreement data. The proportions of runs of various lengths are plotted. The theoretical histogram was generated with $p(X)=.295$ and Formula 2. The observed histogram was generated by summing the number of disagreement runs of each length and then dividing each sum by the total number of runs. The result suggests that there is a lower proportion of disagreement runs of 1 unit and a higher proportion of dis-
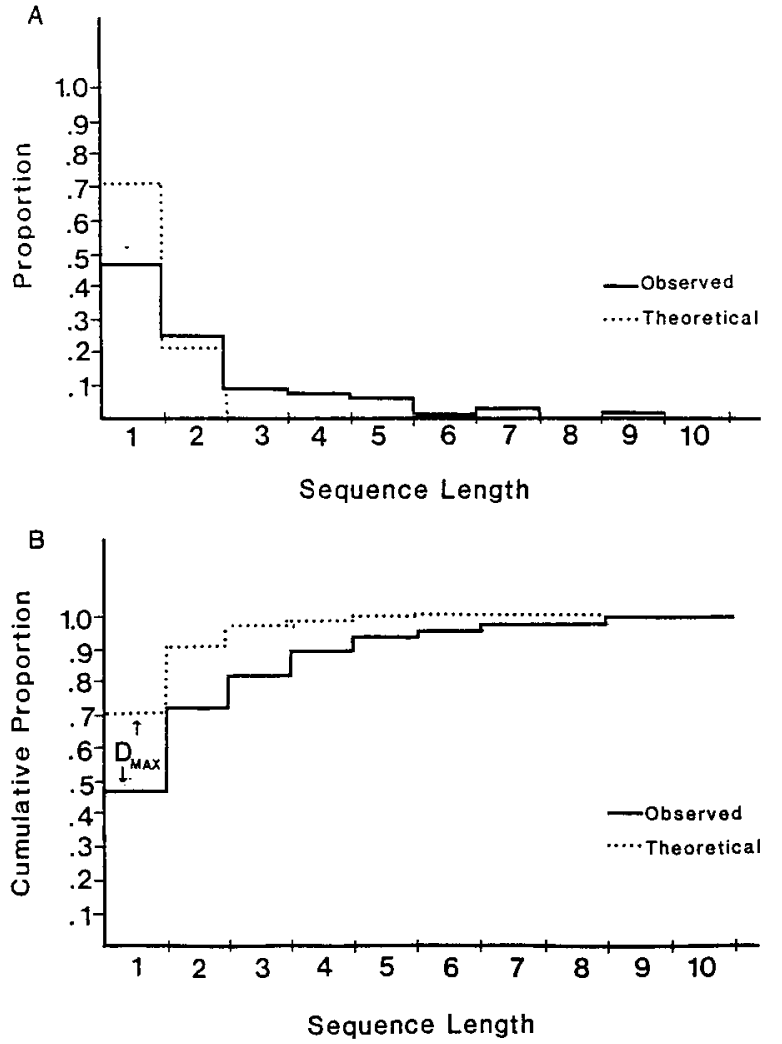

Figure 1. Observed and theoretical histograms for sequences of disagreements: $\mathbf{A}=$ proportion; $\mathbf{B}=$ cumulative proportion.

agreement runs of 2-5 units than would be expected by chance. Figure 1, Panel B, provides cumulative frequency histograms that are used to locate the maximum difference in the distributions $(D \max )$.

The Kolmogorov-Smirnov test is performed on $D \max$, the value of which is compared to a critical value $(1.36 / N$ for $p<.05$, where $N$ is the number of runs used to generate the observed histogram). When $D$ max exceeds the critical value, the null hypothesis that the observed histogram was derived from some theorized process (in this case a Bernoulli process) is rejected (see Siegel, 1956, pp. 47-52).

For this sample of disagreements, $D \max =.235$, and the critical value is .115 for $p<.05$, given 140 runs total. (When the procedure is repeated with the bounds of the $95 \%$ confidence interval of the estimate, $D \max$ for the lower bound is .261 and for the upper bound .207 , both $p s<.05$.) Therefore, the distribution of disagreement runs is unlikely to have been generated by chance.

The same $Z$ formula that is used to calculate the lagsequential statistic (see Allison \& Liker, 1982) can be used as a post hoc test for the Kolmogorov-Smirnov test. It is applied in a manner similar to that which Bakeman (1983) suggested for examining the lagged relationship between a joint event (e.g., the event where $A$ is followed directly by $B$ in a series) and another event $(C)$. The joint event, in this case $A B$, is considered to constitute a distinct event. Base-rate probabilities are determined for the joint event, 
and a $Z$ score for the occurrence of $(A B) C$ is computed as before.

Using this approach, it is possible to determine whether the second turn of a run is more likely than chance, given that a run beginning has occurred (i.e., the joint event, non $X$, followed by $X$ ). The procedure may be repeated to determine whether the third turn of a run is more likely than chance, given that a run of two turns has occurred (i.e., the joint event, non $X, X, X)$. It may be repeated to determine whether the fourth turn of a run is more likely than chance, given knowledge of the first three turns, and so forth.

These iterative $Z$ tests are independent of one another. They should be applied only to the subset of run lengths on which cumulative distributions differ and that also contain at least 20 instances of the necessary joint event. The result reveals the particular run lengths that distinguish the events of interest from what would be expected to occur by chance. (Step-by-step calculations are presented in Appendix A.)

This procedure was performed on the disagreement runs in the sample data to examine run lengths of 2-7. All $Z$ scores exceeded the critical value: $7.91 \leq Z \leq 16.58$. Thus, disagreements tend to occur in runs of those lengths at a substantially higher frequency than chance. Note that when the adjusted probability of the occurrence of a disagreement is very low or high, it is necessary to evaluate the $Z$ score with respect to the skewed distribution that obtains.

\section{Step 2: Determining Whether Disagreement Runs Cluster Together}

The sample data suggest that disagreement runs tend to occur in proximity to one another. This hypothesis is evaluated by examining the distribution of runs of nondisagreements. If disagreement runs do tend to occur in proximity to one another, there will be an inflated proportion of brief nondisagreement runs because they separate disagreement runs that are close together.

After we extract information about runs of disagreements, each run is collapsed to a single disagreement, and the total is adjusted accordingly. (The sample data contain 304 disagreements in 140 runs. Thus, 164 [304-140] is subtracted from the total number of units in the trimmed series.) We know that each run except the last is followed by a nondisagreement. Thus, these nondisagreements also are subtracted from the total (139 in this sample data). The base-rate probability of the occurrence of a nondisagreement after any event equals the adjusted total minus one (the last element in the series cannot follow any other element) minus the number of disagreement runs divided by the adjusted total minus one (.808 for the sample data).

The frequency histogram for nondisagreements that was observed and the one that would be expected to occur by chance are shown in Panel A of Figure 2. Note that the theoretical histogram predicts a much lower proportion of runs of Length 1 than was actually obtained. Cumula-
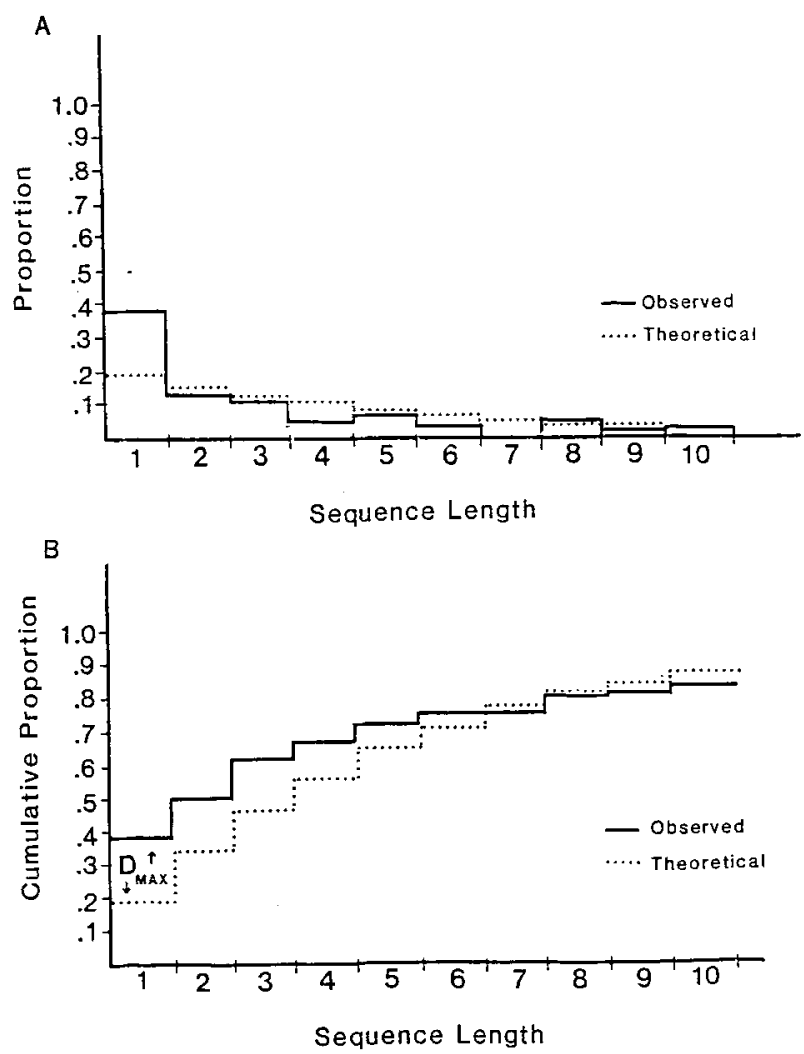

Figure 2. Observed and theoretical histograms for sequences of nondisagreements: $\mathbf{A}=$ proportion; $\mathrm{B}=$ cumulative proportion.

tive frequency histograms were constructed (Figure 2, Panel B), Dmax $=.189$, the critical value for $p=.01$ is .138 , given 139 runs total. (For the upper and lower bounds of the $95 \%$ confidence interval, $D \max$ is .215 and .163 , respectively.) Therefore, the distributions are different $(p<.01)$.

To determine which runs of nondisagreements are likely to occur between disagreement runs, iterative $Z$ scores are performed. Some alterations in the calculations are necessary (see Appendix B). The $Z$ scores for Lengths $1-4$ are: $-6.90,-1.75,-1.59$, and 1.45 . Thus, the proportion of runs of Length 1 is much greater than chance $(p<.01)$, and the proportion of runs of Length 2 are marginally greater than chance $(p<.05)$.

\section{Step 3: Examining Runs of Disagreements Containing Intervening Singleton Nondisagreements}

Disagreement runs appear to "arc over" singleton nondisagreements. It is possible to examine runs taking this into account, by recalculating the frequency histogram of disagreement lengths, allowing embedded singleton nondisagreements, which are not counted in determining run length. This distribution is compared to a different theoretical distribution using the Kolmogorov-Smirnov test as before. The appropriate theoretical distribution is given by:

$$
\sum_{i=1, \infty} Q^{2} P^{i-1}(1+Q)^{i-1}
$$


This formula was derived by using a model of a Bernoulli process and the binomial theorem. Like Formula 2, it is an infinite, convergent series that sums to 1 .

Figure 3 presents the frequency histograms and cumulative frequency histograms for the observed and theoretical distributions of disagreements, allowing single nondisagreements to come between one or more pairs of disagreements in a run. $D \max =.214$, the cutscore for $p=.01$ is .177 , given 85 runs total. ( $D \max$ for the upper and lower bounds of the $95 \%$ confidence interval is .197 and .231 , respectively.) Therefore, the theoretical histogram of items with the base-rate probability of the disagreements and allowing for embedded, singleton nondisagreements is different from the frequency histogram that was actually obtained.

Post hoc $Z$ tests, like those already described, were performed. The procedure is altered somewhat when an embedded nondisagreement is allowed (Appendix C presents the step-by-step calculations). The run lengths 2-13 were examined, and all yielded $Z$ scores beyond the $p<.01$ level (range: 4.02-7.66). Thus, when embedded singleton nondisagreements are allowed, disagreement runs of at least 13 disagreements occur with greater than chance probability.

\section{Step 4: A Kolmogorov-Smirnov Test for Runs of Nondisagreements Where Runs of Length 1 Have Been Removed}

To determine whether there is more information pertaining to the dispersion of disagreement runs, the distribution of nondisagreements, after deleting runs of Length 1, is compared with the appropriate theoretical distribution. The proportions of runs of various lengths is recomputed with the runs of Length 1 deleted. The new base-rate probability of nondisagreements is calculated as above, except that the embedded nondisagreements are subtracted from the adjusted total (yielding .793 for the sample data). The theoretical distribution of nondisagreements can now be calculated using Formula 3. Simply note that the results of the formula for Length 1 now refer to Length 2, those for Length 2 to Length 3, and so forth.

$D \max =.135$ when the observed and theoretical distributions are compared, and the critical value for $p=.05$ is . 148 , given 84 runs total. Thus, the distribution of nondisagreements with runs of Length 1 removed conforms to what would be expected to occur under chance conditions. This result gives the endpoint for an examination of the dispersion of disagreements in this conversation.

\section{Summary of Results Pertaining to Disagreement Runs}

Arguments are constructed by immediately replying in disagreement to another's disagreement. Failure to reply within a few turns terminates an argument. While some disagreements do not receive a reply, most do. Furthermore, the replies tend to receive replies, and so forth. Thus, in the sample conversation, disagreements are generative of other disagreements.
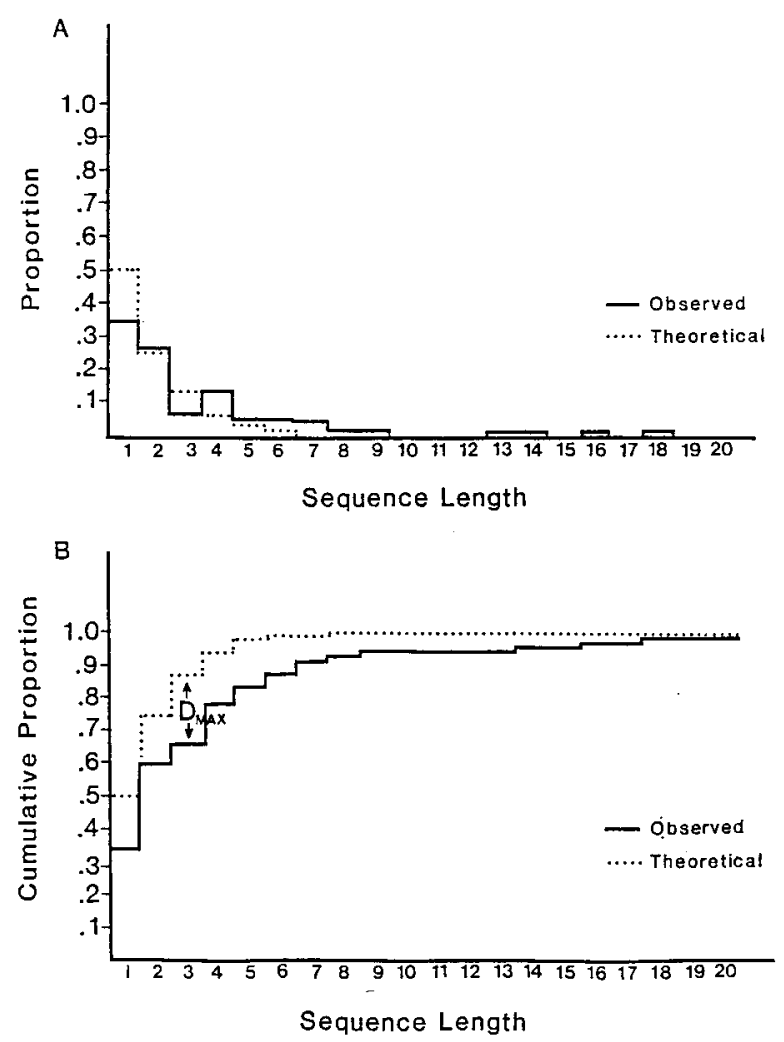

Figure 3. Observed and theoretical histograms for disagreement sequences including intervening singleton nondisagreements: $\mathbf{A}=$ proportion; $\mathbf{B}=$ cumulative proportion.

Surveying the overall distribution of disagreements in the sample conversation and knowing that $41.9 \%$ of the turns of talk in the sample conversation consisted of disagreements allows the description to be expanded. The sample conversation was quite argumentative in tone and substance; it contained continual brief arguments and frequently occurring sustained argument. Such a conclusion is consistent with my experience as an observer of the sample conversation, if the conclusion is qualified by the additional fact that the tone of that conversation was also markedly playful.

\section{RUNS OF STORY CONTINUATIONS IN THE SAME FIFTH GRADERS' CONVERSATION}

A story continuation is a turn at talk in which the speaker explicitly forwards the narration of an incident which has already been introduced into the conversation. The story continuations examined are those in the same conversation of fifth graders examined above.

\section{Step 1: Examining the Distribution of Runs of Story Continuations}

The frequency histogram for story continuations and the one that would be expected by chance are given in Panel A of Figure 4, and the cumulative frequency histograms are provided in Panel B. D $\max =.050$, which is 

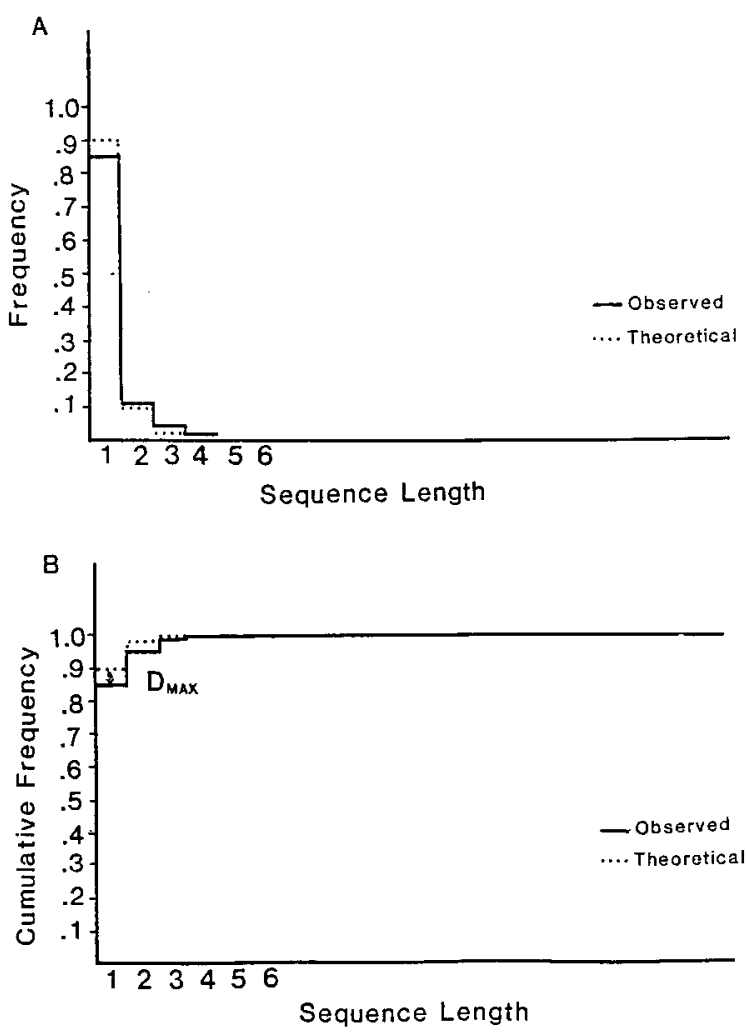

Figure 4. Observed and theoretical histograms for story continuations: $A=$ frequency; $B=$ cumulative frequency.

far less than the cutscore for $p=.05$ of .147 , given 86 runs total. The distribution of runs of story continuations closely conforms to chance expectations. Nearly all story continuations occur singly in conversation.

\section{Step 2: Examining the Cohesiveness of Story-Continuation Runs}

The frequency histogram of nonstory continuations and the one that would be expected by chance are presented in Figure 5, Panel A, and the cumulative frequency histograms are presented in Panel B. The substantial difference between the two histograms is evident in Panel B. $D \max =.189$ and the critical value for $p=.01$ is .177 , given the total of 85 runs. (The upper bound of the $95 \%$ confidence interval of the estimated probability of nonstory continuations and the lower bound yield $D \max$ of .206 and .161, respectively.) Thus, the frequency histogram for nonstory continuations is not what would be expected by chance.

Post hoc $Z$ scores reveal that nonstory-continuation runs of Lengths 1-4 occur more frequently than would be expected $(-2.61 \leq Z \leq-7.33)$. Thus, although story continuations do not form runs in a manner different from what is expected by chance, story-continuation runs do occur near each other.

\section{Step 3: Examining Runs of Story Continuations with Embedded Nonstory Continuations}

Next, the observed frequency histogram of story continuations allowing for singleton embedded nonstory con- tinuations is compared with the theoretical histogram generated with Formula 3 using $p(X)=101$. $D \max =.090$, where the critical value for $p=.05$ is .161 , given 71 sequences. The frequency distribution of story continuations allowing for singleton nonstory continuations is not different from what is expected by chance.

Since the proportion of nonstory-continuation runs of Length 2 is also greater than is expected by chance, the frequency histogram of story-continuation runs allowing up to 2 intervening nonstory continuations is compared to the frequency histogram that is expected by chance. The formula for the theoretical distribution is given by the following equation:

$$
\begin{gathered}
\sum_{i=1, \infty} Q^{3} P^{i-1}\left[(1+Q)^{i-1}+\left(1+Q^{2}\right)^{i-1}-\right. \\
1+\sum_{j=1, i-2} C_{j}^{i-1} Q^{2 j} Q^{i-j-1}+ \\
\left.\sum_{j=1, i-3} C_{j}^{i-1}\left(\sum_{k=1, i-j-2} C_{k}^{i-j-1} Q^{2 k} Q^{i-j-k-1}\right)\right] .
\end{gathered}
$$

Like Formulas 2 and 3, it is an infinite, convergent series that sums to $1 . D \max =.113$, where the critical value for $p=.05$ is .177 , given 59 runs. Thus, storycontinuation runs containing up to two embedded nonstory continuations are not different from what is expected by chance.

It is possible to repeat this process allowing up to three embedded nonstory continuations. The formula for the
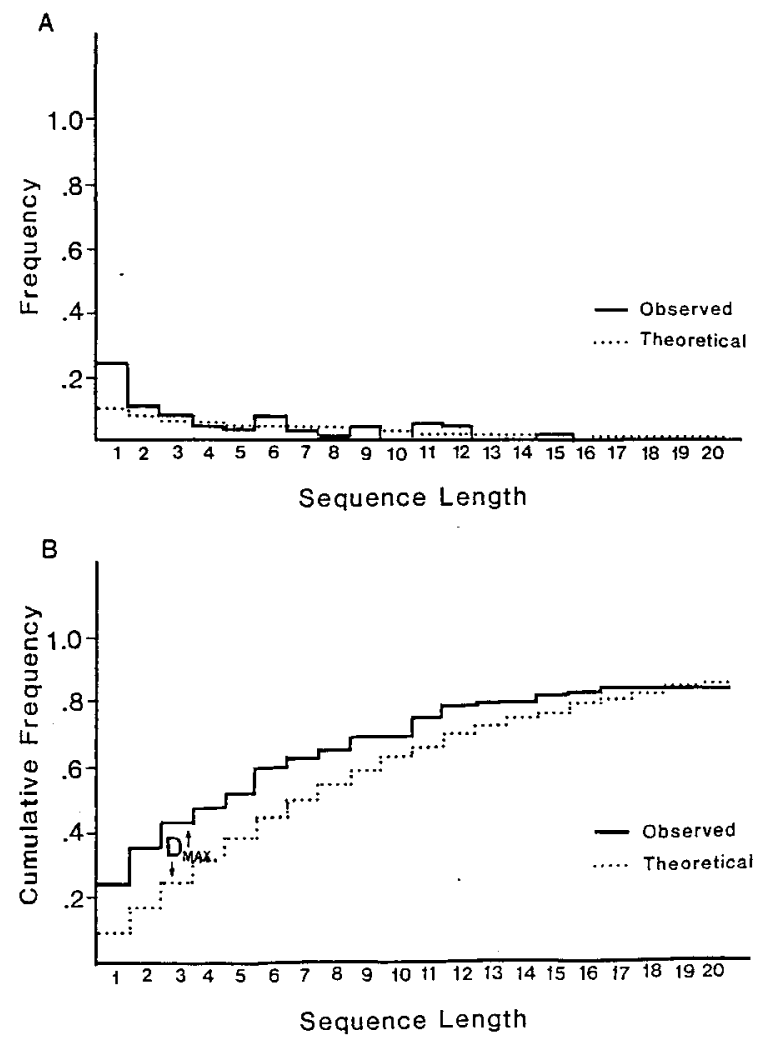

Figure 5. Observed and theoretical histograms for nonstory continuations: $A=$ frequency; $B=$ cumulative frequency. 
theoretical distribution becomes forbidding, but a good approximation can be obtained by using a modeling program. This procedure was followed, yielding a $D \max =$ .121 where the $p=.05$ critical value is .189 . A nonsignificant result was also obtained when up to four intervening nonstory continuations were permitted in runs of story continuations. Thus, there is no evidence to suggest that story continuations alone form runs, but they do tend to occur near to each other in conversation.

\section{Summary of Results Pertaining to Story-Continuation Runs}

The sample conversation contains segments of talk that are bounded by story continuations. Thus, a portion of the conversation consists of members' orienting to events that are narrated. Since runs of story continuations with embedded elements typically contain two or three narrations in addition to the story's beginning and account for $32.5 \%$ of the sample conversation, storytelling sequences are brief but occur frequently.

\section{DISCUSSION}

A method for determining the run structure of events in a series has been presented and illustrated with one conversation generated by a small group of fifth graders. Disagreements and story continuations in this conversation were examined and found to display different run structures. Together they account for a substantial portion of the conversation, $73 \%$ with adjustment for overlap. The sample conversation consisted largely of good-natured arguments interspersed with storytelling.

The sequencing of other kinds of turns could be examined and then reconciled with that of disagreements and story continuations to account for even more of the sample conversation's organization. Thus, the method of runs analysis described in this paper can be employed repeatedly on the same series of codes to accumulate information on the overall run structure.

\section{REFERENCES}

Allison, P. D., \& LIKER, J. K. (1982). Analyzing sequential data on dyadic interaction: A comment on Gottman. Psychological Bulletin, 91, 393-403.

Als, H., Tronick, E., \& Brazelton, T. B. (1979). Analysis of faceto-face interaction in infant-adult dyads. In M. E. Lamb, S. J. Suomi, \& G. R. Stephenson (Eds.), Social interaction analysis (pp. 33-76). Madison, WI: University of Wisconsin Press.

BaKEMAN, R. (1983). Computing the lag sequential statistics: The ELAG program. Behavior Research Methods \& Instrumentation, 15, 530-535.

BaKeman, R., \& Brown, J. V. (1977). Behavioral dialogs: An approach to the assessment of mother-infant interaction. Child Development, 48, 195-203.

BAKEMAN, R., \& BRownLEE, J, R. (1982). Social rules governing object conflicts in toddlers and preschoolers. In K. H. Rubin \& H. S. Ross (Eds.), Peer relationships and social skills in childhood (pp. 99 112). New York: Springer.

Brownlee, J. R., \& Germond, J. (1984, April). Dyadic styles of interaction between mothers and infants of varying characteristics. Paper presented at the biennial meeting of the Southwestern Society for Research in Human Development, Denver.
Dorval, B., \& EcKerman, C. O. (1984). Developmental trends in the quality of conversation achieved by small groups of acquainted peers. Monographs of Society for Research in Child Development (No. 206).

DRAKE, A. W. (1967). Fundamentals of applied probability theory. New York: McGraw-Hill.

Eckerman, C. O., \& Stein, M. R. (1982). The toddler's emerging interactive skills. In K. H. Rubin \& H. S. Ross (Eds.), Peer relationships and social skills in childhood (pp. 41-72). New York: Springer.

Goldman, B. D., \& Ross, H. S. (1978). Social skills in action: The analysis of early peer games. In J. Glick \& K. A. Clarke-Stewart (Eds.), The development of social understanding (pp. 177-212). New York: Gardner Press.

Gotrman, J. M. (1979). Marital interaction. New York: Academic Press.

Gottman, J. M. (1983). How children become friends. Monographs of Society for Research in Child Development (No. 201).

GotTman, J. M., \& Bakeman, R. (1979). The sequential analysis of observational data. In M. E. Lamb, S. J. Suomi, \& G. R. Stephenson (Eds.), Social interaction analysis (pp. 185-206). Madison, WI: University of Wisconsin Press.

Patterson, G. R. (1980). Mothers: The unacknowledged victims. Monographs of Society for Research in Child Development (No. 186).

SACKETT, G. P. (1977). Lag sequential analysis. In G. P. Sackett (Ed.), Observing behavior (Vol. 2). Baltimore, MD: University Park Press.

SACKetT, G. P. (1979). The lag sequential analysis of contingency and cyclicity in behavior interaction research. In J. Osofsky (Ed.), Handbook of infant development. New York: Wiley.

SACKETT, G. P. (1980). Lag sequential analysis as a data reduction technique in social interaction research. In D. Sawin, R. Hawkins, L. Walker, \& J. Penticuff (Eds.), Exceptional infant (Vol. 4). New York: Bruner-Mazel.

SIEGEL, S. (1956). Nonparametric statistics. New York: McGraw-Hill. Suomi, S. J. (1979). Levels of analysis for interactive data collected on monkeys living in complex social groups. In M. E. Lamb, S. J. Suomi, \& G. R. Stephenson (Eds.), Social interaction analysis (pp. 119-136). Madison, WI: University of Wisconsin Press.

\section{APPENDIX A \\ Rules for $Z$ Tests Subsequent to a Kolmogorov-Smirnov Test}

1. Let us define $X$ as the event of interest. The following is a series of rules for determining whether runs of $X s$ of Length $L$ are more likely than chance to include another $X$ in place $L+1$, given knowledge of the runs of $X \mathrm{~s}$ of Length $1-L$.

These rules should be applied iteratively, beginning with $L=1$. Strictly speaking, the iteration should be continued until the frequency of free $X s$ is low $(<20)$. However, it seems that once there are nonsignificant results for a few trials, the subsequent ones are likely to be nonsignificant as well.

2. Trim the series so that it is bounded by runs of $X s$ of Length $L$ or greater. On the right end, save the item directly following the criterion run.

Adjust the total frequency and the frequency of $X s$ accordingly. Add 1 to the total frequency, because the first run, by definition, is preceded by a non $X$.

3. Sequences of $X s$ that are shorter than $L$ are collapsed to a single non $X$. As the result of preceding computations, we know that each is bounded by non $X$ s. So, the shorter $X$ runs and a non $X$ from both ends of each run are removed. Since each sequence of $X \mathrm{~s}$ shorter than $L$ is replaced by a single non $X$, one can imagine removing each run and only one of the nonXs that bound that run.

Adjust the total frequency and the frequency of Xs accordingly. Since the procedure is performed iteratively, one can 


\section{Appendix A (Continued)}

simply remove the runs of Length $L-1$, as soon as $L$ has been incremented, to begin the next iteration.

4. Since we examine all runs of $X s$ of Length $L$ or greater, the leading non $X$ and the following $L X \mathrm{~s}$ are collapsed into a single criterion event. Thus, total number of events and the number of $X \mathrm{~s}$ are both adjusted by subtracting $L$ for each criterion run.

5. The probability of $X$ for this test is the adjusted total of $X$ s divided by the adjusted total number of turns.

6. The conditional probability of $X$, given a prior run of $X \mathrm{~s}$ of Length $L$, equals the number of runs of Length $L+1$ or longer divided by the number of Length $L$ or longer.

7. The conditional probability of $X$, given no prior run of $X \mathrm{~s}$ of Length $L$, equals the adjusted total of $X$ s minus the $X \mathrm{~s}$ in the numerator of Step 6 divided by the adjusted total turn minus the denominator in Step 6.

8. The following is a $Z$ test to evaluate the equivalence of the two conditional probabilities computed in Steps 6 and 7 , where $X$ seq refers to runs of Length $L$ and $n(X)$ refers to the adjusted total of $X s$ :

$$
Z=\frac{p\left(X \mid X_{\text {seq }}\right)-p(X \mid \text { nonXseq })}{\sqrt{p(X) \cdot q(X) \cdot\left[\frac{1}{n\left(X_{\text {seq }}\right.}+\frac{1}{n(\text { nonXseq })}\right]}}
$$

\section{APPENDIX B}

Rules for $Z$ Tests Subsequent to a Kolmogorov-Smirnov Test for Nondisagreement Runs

1. Same as in Appendix A, except that $X$ now refers to a nondisagreement.

\section{Appendix B (Continued)}

2. Before performing the operations described in Appen$\operatorname{dix} \mathrm{A}$, each run of disagreements, including the nondisagreements that bound it, is collapsed into a single disagreement. This is done because information about runs of disagreements has already been extracted from the series. Totals are adjusted accordingly.

Steps 3-8 are the same as in Appendix A.

\section{APPENDIX C \\ Rules for $Z$ Tests Subsequent to a \\ Kolmogorov-Smirnov Test for Disagreement \\ Runs Allowing One Nondisagreement}

1-4. Same as in Appendix A.

5. Count the number of nonXs in runs of Length $2-L$. Remove that number from the adjusted total. Also, count the number of runs of Length $L+$ which have a non $X$ immediately after the $L$ th $X$, and subtract that number from the adjusted total.

6. The probability of $X$ for this test is the adjusted total of $X s$, divided by the adjusted total number of turns. We are interested in the probability that the run will be continued which can occur in two ways: an $X$ occurring immediately or a non $X$ followed by an $X$. Thus, the probability of $L+$ : $P(L+)=P(X)+P($ non $X) * P(X)$.

7-9. Same as in Appendix A, Steps 6-8.

(Manuscript received June 14, 1985; revision accepted for publication February 18, 1986) 